\title{
Visible Points on Modular Exponential Curves
}

by

\author{
Tsz Ho CHAN and Igor E. SHPARLINSKI
}

Presented by Jerzy KACZOROWSKI

Summary. We obtain an asymptotic formula for the number of visible points $(x, y)$, that is, with $\operatorname{gcd}(x, y)=1$, which lie in the box $[1, U] \times[1, V]$ and also belong to the exponential modular curves $y \equiv a g^{x}(\bmod p)$. Among other tools, some recent results of additive combinatorics due to J. Bourgain and M. Z. Garaev play a crucial role in our argument.

1. Introduction. We consider points on the exponential modular curves

$$
\mathcal{E}_{a, g, p}=\left\{(x, y): y \equiv a g^{x}(\bmod p)\right\} .
$$

Furthermore, for real $U$ and $V$ we use $\mathcal{E}_{a, g, p}(U, V)$ to denote the set of points $(x, y) \in \mathcal{E}_{a, g, p}$ which lie in the box $[1, U] \times[1, V]$.

Here we obtain an asymptotic formula for the number $N_{a, g, p}(U, V)$ of visible points $(x, y) \in \mathcal{E}_{a, g, p}(U, V)$, that is, points satisfying $\operatorname{gcd}(x, y)=1$.

We note that visible points on some other curves have been studied in [10, 11, 12]. However, their methods do not extend to the points on $\mathcal{E}_{a, g, p}$. In fact, a result of [2] is a crucial ingredient of our argument.

Throughout the paper, the implied constants in the symbols " $O$ " and "«" are absolute (we recall that $A=O(B)$ and $A \ll B$ are both equivalent to the inequality $|A| \leq c B$ with some constant $c>0$ ).

TheOREM 1. For $(a, p)=1$ and any primitive root $\vartheta$ modulo $p$,

$$
N_{a, \vartheta, p}(U, V)=\frac{6}{\pi^{2}} \cdot \frac{U V}{p}+O\left(\left(\frac{U^{1 / 2} V^{1 / 2}}{p^{1 / 4}}+\frac{U}{V^{1 / 35}}+\frac{V}{U^{1 / 35}}\right) p^{o(1)}\right)
$$

for $1 \leq U, V \leq p-1$ with $U V \geq p^{3 / 2}$. 
Note that the bound of Theorem 1 is nontrivial if $\min \{U, V\} \geq p^{35 / 36+\varepsilon}$ for some fixed $\varepsilon>0$.

2. Preparations. The following estimate is very well known ([7, 9] and also [3, 4, 6, 8]).

Let $M_{a, g, p}(U, V)$ be the number of points $(x, y) \in \mathcal{E}_{a, g, p}(U, V)$.

Lemma 2. For $(a g, p)=1$ and $U, V \leq t$ where $t$ is the multiplicative order of $g$ modulo $p$,

$$
M_{a, g, p}(U, V)=\frac{U V}{p}+O\left(p^{1 / 2}(\log p)^{2}\right) .
$$

We now present an upper bound on $M_{a, g, p}(U, V)$ which is better than that in Lemma 2 for small $U$ and $V$.

Lemma 3. For $(a g, p)=1$ and $U, V \leq t$ where $t$ is the multiplicative order of $g$ modulo $p$, we have

$$
M_{a, g, p}(U, V) \ll \frac{U V}{p}+\frac{V}{U^{1 / 11+o(1)}}+\frac{U}{V^{1 / 11+o(1)}}
$$

as $U, V \rightarrow \infty$.

Proof. By [2, Corollary 5], we have

$$
M_{a, g, p}(U, U) \ll \frac{U^{2}}{p}+\frac{U}{U^{1 / 11+o(1)}} .
$$

For $V \geq U$, we just divide the rectangle into $O(V / U)$ squares with side length $U$. Then

$$
M_{a, g, p}(U, V) \ll \frac{V}{U}\left(\frac{U^{2}}{p}+\frac{U}{U^{1 / 11+o(1)}}\right),
$$

which gives the desired estimate. The proof for $U \geq V$ is similar.

We denote by $R_{a, g, p}(K ; D)$ the number of solutions to the congruence

$$
a d \equiv g^{d}(\bmod p), \quad K+1 \leq d \leq K+D .
$$

Lemma 4. For $(a g, p)=1$ and $D \leq p$, we have

$$
R_{a, g, p}(K ; D) \ll D^{1 / 2} .
$$

Proof. Clearly $R_{a, g, p}(K ; D)^{2}$ is equal to the number of solutions to the system of congruences

$$
a d \equiv g^{d}(\bmod p) \quad \text { and } \quad a f \equiv g^{f}(\bmod p), \quad K+1 \leq d, f \leq K+D .
$$

Thus writing $f=d+e$ we see that

$$
R_{a, g, p}(K ; D)^{2} \leq Q_{a, g, p}(K ; D),
$$


where $Q_{a, g, p}(K ; D)$ is the number of solutions to the system of congruences

$$
a d \equiv g^{d}(\bmod p) \quad \text { and } \quad a(d+e) \equiv g^{d+e}(\bmod p),
$$

where

$$
-D<e<D \quad \text { and } \quad K+1 \leq d \leq K+D .
$$

We see that the above congruences imply

$$
e \equiv d\left(g^{e}-1\right)(\bmod p) .
$$

For every $e$ with $g^{e} \not \equiv 1(\bmod p)$ the congruence $(2)$ defines $d$ uniquely, so there are $O(D)$ such solutions $(e, d)$. For $g^{e} \equiv 1(\bmod p)$ we see from (2) that $e \equiv 0(\bmod p)$, which in turn implies $e=0$ (and $d$ can take any values with $K+1 \leq d \leq K+D)$; so again there are $O(D)$ such solutions $(e, d)$. Therefore

$$
Q_{a, g, p}(K ; D) \ll D,
$$

and recalling (1) we conclude the proof.

3. Proof of Theorem 1. For $(a, p)=1=(\vartheta, p)$, we have $\left(a \vartheta^{y}, p\right)=1$. By the inclusion-exclusion principle,

$$
\begin{aligned}
N_{a, \vartheta, p}(U, V) & =\sum_{\substack{d=1 \\
\operatorname{gcd}(d, p)=1}}^{\infty} \mu(d) \sum_{\substack{(x, y) \in \mathcal{E}_{a, \vartheta, p}(U, V) \\
d \mid(x, y)}} 1 \\
& =\sum_{\substack{d=1 \\
\operatorname{gcd}(d, p)=1}}^{\infty} \mu(d) \sum_{1 \leq u \leq U / d} \sum_{\substack{1 \leq v \leq V / d \\
d v \equiv a \vartheta^{d u} \\
(\bmod p)}} 1 \\
& =\sum_{\substack{d=1 \\
\operatorname{gcd}(d, p)=1}}^{\infty} \mu(d) M_{a \bar{d}, \vartheta^{d}, p}\left(\frac{U}{d}, \frac{V}{d}\right),
\end{aligned}
$$

where $\bar{d}$ is the multiplicative inverse of $d$ modulo $p$ and $\mu(d)$ is the Möbius function (see [5, Section 16.3]). We now choose two real parameters $p \geq \Delta>$ $\delta \geq 1$ and write

$$
N_{a, \vartheta, p}(U, V)=\Sigma_{1}+\Sigma_{2}+\Sigma_{3},
$$

where

$$
\begin{aligned}
& \Sigma_{1}=\sum_{\substack{\operatorname{gcd}(d, p)=1 \\
1 \leq d \leq \delta}} \mu(d) M_{a \bar{d}, \vartheta^{d}, p}\left(\frac{U}{d}, \frac{V}{d}\right), \\
& \Sigma_{2}=\sum_{\substack{\operatorname{gcd}(d, p)=1 \\
\delta<d \leq \Delta}} \mu(d) M_{a \bar{d}, \vartheta^{d}, p}\left(\frac{U}{d}, \frac{V}{d}\right),
\end{aligned}
$$




$$
\Sigma_{3}=\sum_{\substack{\operatorname{gcd}(d, p)=1 \\ d>\Delta}} \mu(d) M_{a \bar{d}, \vartheta^{d}, p}\left(\frac{U}{d}, \frac{V}{d}\right) .
$$

We use Lemmas 2, 3 and 4 to estimate $\Sigma_{1}, \Sigma_{2}$ and $\Sigma_{3}$ respectively.

By Lemma 2,

$$
\begin{aligned}
\Sigma_{1} & =\sum_{d \leq \delta} \mu(d)\left(\frac{U}{d} \cdot \frac{V}{d} \cdot \frac{1}{p}+O\left(p^{1 / 2}(\log p)^{2}\right)\right) \\
& =\frac{U V}{p} \sum_{d \leq \delta} \frac{\mu(d)}{d^{2}}+O\left(\delta p^{1 / 2}(\log p)^{2}\right) \\
& =\frac{6}{\pi^{2}} \cdot \frac{U V}{p}+O\left(\frac{U V}{p \delta}+\delta p^{1 / 2}(\log p)^{2}\right)
\end{aligned}
$$

since

$$
\sum_{d=1}^{\infty} \frac{\mu(d)}{d^{2}}=\frac{1}{\zeta(2)}=\frac{6}{\pi^{2}}
$$

(see [5, Equation (17.2.2)]).

Without loss of generality, we can assume that $V \geq U$. Then by Lemma 3 ,

$$
\begin{aligned}
\Sigma_{2} & \ll \sum_{\delta<d \leq \Delta}\left(\frac{U V}{d^{2} p}+V U^{-1 / 11} d^{-10 / 11} p^{o(1)}\right) \\
& \ll \frac{U V}{p \delta}+V U^{-1 / 11} \Delta^{1 / 11} p^{o(1)} .
\end{aligned}
$$

We now define an integer $L$ by the inequalities

$$
2^{L} \Delta<\min (U, V) \leq 2^{L+1} \Delta
$$

and write

$$
\begin{aligned}
& \Sigma_{3} \leq \sum_{i=0}^{L} \sum_{2^{i} \Delta<d \leq 2^{i+1} \Delta} M_{a \bar{d}, \vartheta^{d}, p}\left(\frac{U}{2^{i} \Delta}, \frac{V}{2^{i} \Delta}\right) \\
& =\sum_{i=0}^{L} \sum_{u \leq \frac{U}{2^{i} \Delta}} \sum_{v \leq \frac{V}{2^{i} \Delta}} \sum_{\substack{2^{i} \Delta<d \leq 2^{i+1} \Delta \\
d \equiv a \bar{v} \vartheta d u(\bmod p)}} 1 .
\end{aligned}
$$

Thus by Lemma 4 .

$$
\Sigma_{3} \ll \sum_{i=0}^{L} \sum_{u \leq \frac{U}{2^{i} \Delta}} \sum_{v \leq \frac{V}{2^{i} \Delta}}\left(2^{i} \Delta\right)^{1 / 2} \ll U V \Delta^{-3 / 2} .
$$

Substituting the above estimates in (3), we obtain 


$$
\begin{aligned}
N_{a, \vartheta, p}(U, V) & -\frac{6}{\pi^{2}} \cdot \frac{U V}{p} \\
& \ll \frac{U V}{p \delta}+\delta p^{1 / 2+o(1)}+V U^{-1 / 11} \Delta^{1 / 11} p^{o(1)}+U V \Delta^{-3 / 2} .
\end{aligned}
$$

We now choose

$$
\delta=U^{1 / 2} V^{1 / 2} p^{-3 / 4}
$$

to balance the first and the second terms and

$$
\Delta=U^{24 / 35}
$$

to balance the third and the fourth terms on the right hand side of (4) (and note that since $U V \geq p^{3 / 2}$ and $U \leq V<p$, the condition $p \geq \Delta>\delta \geq 1$ is satisfied), getting

$$
N_{a, \vartheta, p}(U, V)-\frac{6}{\pi^{2}} \cdot \frac{U V}{p} \ll\left(U^{1 / 2} V^{1 / 2} p^{-1 / 4}+V U^{-1 / 35}\right) p^{o(1)},
$$

which gives the desired result.

4. Comments. We remark that Lemma 3, which in turn depends on some results of additive combinatorics due to J. Bourgain and M. Z. Garaev [1], is an essential ingredient of our proof. Just a combination of Lemmas 2 and 4 is not sufficient to derive an asymptotic formula for $N_{a, \vartheta, p}(U, V)$. On the other hand, the ingredients of this paper are quite sufficient to obtain an asymptotic formula for $N_{a, g, p}(U, V)$ also in the case when $g$ is not necessarily a primitive root modulo $p$.

\section{References}

[1] J. Bourgain and M. Z. Garaev, On a variant of sum-product estimates and explicit exponential sum bounds in prime fields, Math. Proc. Cambridge Philos. Soc. 146 (2008), 1-21.

[2] T. H. Chan and I. E. Shparlinski, On the concentration of points on modular hyperbolas and exponential curves, Acta Arith. 142 (2010), 59-66.

[3] C. Cobeli, S. Gonek and A. Zaharescu, On the distribution of small powers of a primitive root, J. Number Theory 88 (2001), 49-58.

[4] M. Z. Garaev, On the logarithmic factor in error term estimates in certain additive congruence problems, Acta Arith. 124 (2006), 27-39.

[5] G. H. Hardy and E. M. Wright, An Introduction to the Theory of Numbers, The Clarendon Press, Oxford Univ. Press, New York, 1979.

[6] S. V. Konyagin and I. E. Shparlinski, Character Sums with Exponential Functions and Their Applications, Cambridge Univ. Press, Cambridge, 1999.

[7] N. M. Korobov, On the distribution of digits in periodic fractions, Mat. Sb. 89 (1972), 654-670 (in Russian).

[8] H. L. Montgomery, Distribution of small powers of a primitive root, in: Advances in Number Theory, Clarendon Press, Oxford, 1993, 137-149. 
[9] H. Niederreiter, Quasi-Monte Carlo methods and pseudo-random numbers, Bull. Amer. Math. Soc. 84 (1978), 957-1041.

[10] I. E. Shparlinski, Primitive points on a modular hyperbola, Bull. Polish Acad. Sci. Math. 54 (2006), 193-200.

[11] I. E. Shparlinski and J. F. Voloch, Visible points on curves over finite fields, ibid. 55 (2007), 193-199.

[12] I. E. Shparlinski and A. Winterhof, Visible points on multidimensional modular hyperbolas, J. Number Theory 128 (2008), 2695-2703.

Tsz Ho Chan

Department of Mathematical Sciences

University of Memphis

Memphis, TN 38152, U.S.A.

E-mail: tchan@memphis.edu
Igor E. Shparlinski Department of Computing Macquarie University Sydney, NSW 2109, Australia E-mail: igor@comp.mq.edu.au

Received December 8, 2009;

received in final form February 26, 2010 